\title{
Significant regression of carotid artery stenosis after treatment with proprotein convertase subtilisin/ kexin type 9 serine protease inhibitor
}

\begin{abstract}
Carotid artery stenosis is one of the major causes of ischemic stroke. Optimal medical treatment includes controlling diabetes, hypertension, and hyperlipidemia, smoking cessation, and anti-platelets to slow down the atherosclerotic plaque progression and are important in secondary stroke prevention. Carotid endarterectomy (CEA) or carotid artery stenting (CAS) is the standard of care for patients with symptomatic carotid artery stenosis $>70 \%$ who has a perioperative stroke or mortality rate $<6 \%$. To date, there has been no report on medical revascularization of carotid artery stenosis. Here we report a case of a 94-year-old patient with asymptomatic $85 \%$ right internal carotid artery (ICA) stenosis, who benefited a 23\% plaque regression after 2 years of evolocumab (Proprotein Convertase Subtilisin/kexin Type 9 Serine Protease Inhibitor) (PCSK-9 inhibitor) injection for her atherosclerotic coronary artery disease. While further studies are necessary, we proposed that PCSK-9 inhibitor could be a safe, promising alternative to CEA or CAS in the revascularization of carotid artery stenosis.
\end{abstract}

Keywords: carotid artery stenosis, pcsk-9 inhibitor, evolocumab, carotid endarterectomy, carotid artery stenting
Volume 7 Issue 5 - 2019

\author{
Nang San Hti Lar Seng, ${ }^{3,4}$ Htin Kyaw, ${ }^{3}$ Nay \\ Min Tun, ${ }^{3}$ Shwe Synn, ${ }^{3}$ Thwe Thwe Tun, ${ }^{3}$ \\ Alexander Myint Swan, ${ }^{3}$ Tracy Than Swan, ${ }^{3}$ \\ Kyaw Hla, ${ }^{3}$ Htun M Aung, ${ }^{3}$ Nway Nway, ${ }^{3}$ May \\ T Zin, ${ }^{3}$ Alexander Myint Swan, ${ }^{1,2,3}$ Cho Cho \\ Khlne, ${ }^{4}$ Win Hlaing, ${ }^{4}$ Khine Wai Wai Soe, ${ }^{4}$ \\ Yadanar Win Lei ${ }^{4}$ \\ 'AOA Professor of MEDICINE, Rutgers New Jersey Medical \\ School, Newark, New Jersey, USA \\ 2President of Garden State Kidney Center, Woodbriydge, USA \\ ${ }^{3}$ Nephrology Hypertension Renal Transplant \& Renal Therapy, \\ NJ 0700I, USA \\ ${ }^{4}$ Duke-NUS Medical School, Singapore
}

Correspondence: Alexander Myint Swan, Department of Medicine, Rutgers New Jersey Medical School 185 S Orange Ave, Newark, NJ 07I03,USA, Email alexmswan@gmail.com

Received: August 24, 2019 | Published: September 20, 2019
Abbreviations: CAS, carotid artery stenting; CEA, carotid endarterectomy; PCSK 9, proprotein convertase subtilisin kexin type 9; RBMC, Raritan bay medical center; ASCD, atherosclerotic coronary artery disease; LDLR, LDL receptors; ICA, internal carotid artery

\section{Introduction}

Cerebrovascular accident is the fifth most common cause of death and the leading cause of disability in the United States. ${ }^{1}$ Extracranial carotid artery stenosis accounts for $8 \%$ of all ischemic stroke. ${ }^{2}$ Carotid artery stenosis is common in the elderly population and reported to be present in about $20 \%$ of people above age $70 .{ }^{3}$ The management of carotid artery stenosis depends on the extent of stenosis and whether the stenosis is symptomatic or asymptomatic. ${ }^{4}$ Carotid artery stenosis is described to be symptomatic when patients experience a transient ischemic attack, amaurosis fugax, or ischemic stroke ipsilateral to the side of stenosis. Medical management of risk factors such as hypertension, diabetes, and hyperlipidemia, anti-platelets, and smoking cessation are recommended for secondary stroke prevention regardless of the extent of stenosis. ${ }^{5}$ Carotid endarterectomy (CEA) is known to prevent long-term stroke risk and is indicated in symptomatic patients with carotid artery stenosis $>70 \%$ who has a perioperative stroke and death risk $<6 \%$. For a patient who has a high surgical risk, carotid artery stenting (CAS) has become a favorable alternative. Studies showed there is no significant difference in the effectiveness and durability of revascularization and risks of perioperative stroke and myocardial infarction between CEA and CAS. ${ }^{6,7,8}$ For asymptomatic carotid artery stenosis, there is no consensus to whether CEA or CAS is indicated. Risks and benefits of either procedure should be carefully analyzed based on patients' comorbidity, life expectancy, and preference. ${ }^{4}$ To date, there is no medical revascularization reported for carotid artery stenosis. Proprotein convertase subtilisin kexin type 9 (PCSK 9) is a protein that binds to LDL-receptors in the hepatocyte and targets them for lysosomal degradation, thereby decreasing the recycling of the LDL receptors to the cell surface. ${ }^{9}$ Evolocumab and alirocumab are the two FDA-approved PCSK-9 inhibitors, indicated in familial hypercholesterolemia and atherosclerotic cardiovascular disease. ${ }^{9}$ By inhibiting PCSK 9, these drugs increase LDL-receptors on the hepatocyte cell surface, thereby lowering circulating LDL level in the blood. In addition, PCSK-9 inhibitors, when used along with a statin, are also reported to cause atheroma regression in patients with coronary artery disease. ${ }^{10}$

\section{Case report}

The patient is a 94-year-old lady who has been following up at our office for primary care since October 2012. Her chronic medical conditions include Type 2 diabetes, hyperlipidemia, and hypertension, for which she was on metformin, linagliptin, atorvastatin, and colesevelam. She had no known history of amaurosis fugax, transient ischemic attack, or stroke. However, on physical examination, she was noted to have right carotid bruit while the rest of the cardiovascular examination was unremarkable. Screening ultrasound showed bilateral plaque in the common carotid arteries in 2012. Her baseline echocardiogram showed mild left ventricular hypertrophy with an estimated ejection fraction of $67 \%$, sclerotic aortic valve, and mild mitral annular calcification. She was started on aspirin $81 \mathrm{mg}$ and clopidogrel $75 \mathrm{mg}$ to prevent cardiovascular and cerebrovascular accidents. In 2014, she was admitted to the hospital (RBMC) for 
dizziness. A carotid ultrasound performed at RBMC showed $85 \%$ stenosis of the right ICA and $<70 \%$ stenosis in the left ICA. At that time, she was recommended to undergo revascularization via CEA or CAS for her right carotid artery stenosis. However, she declined the intervention due to her advanced age and perioperative risks. In view of her atherosclerotic coronary artery disease, we started her on PCSK9 inhibitor (Evolocumab $140 \mathrm{mg}$ ) bi-weekly injection in March 2015. Her lipid profile before and after PCSK-9 inhibitor is shown in Table 1. Interestingly, her right carotid bruit also disappeared after 2 years of evolocumab bi-weekly injection. Carotid ultrasound on September 2017 showed $62 \%$ stenosis in the right ICA and 58\% in left ICA.

\section{Discussion}

While a patient with atherosclerotic heart disease may or may not have carotid artery stenosis, it is highly likely that a patient with carotid artery stenosis has atherosclerotic coronary artery disease (ASCD). This is supported by the excess risk of MI following CEA or CAS, which is also an important cause of perioperative death ${ }^{7}$. While CEA and CAS are intended for stroke prophylaxis, $2.3-4.1 \%$ of patients ended up with perioperative stroke. ${ }^{8}$ Furthermore, even after CEA and CAS are performed, there is still a high risk of severe restenosis $(>70 \%)$ in about $10 \%$ of the patients. ${ }^{11}$ In this case, we started the patient on PCSK-9 inhibitor (evolocumab) in view of her ASCD. Prior to evolocumab injection, her lipid profile was poorly controlled for years despite being on the maximal dose of atorvastatin. Following evolocumab injection, her lipid profile significantly improved (Table 1). Incidentally, we also found that there is a $23 \%$ reduction in her right carotid artery stenosis over the course of 2 years after the PCSK9 inhibitor was initiated. The benefit of using PCSK-9 inhibitor is that it confers a significant lipid-lowering ability and plaque regression. ${ }^{10}$ The side effects of PCSK-9 inhibitor are minimal and include skin irritation at the injection site and nasopharyngitis. ${ }^{12}$ More importantly, unlike CEA and CAS, there is no periprocedural risk of stroke, MI, and death and yet it conferred drastic revascularization of carotid revascularization in our case. ${ }^{13-24}$

Table I Lipid profile before and after PCSK-9 inhibitor injections

\begin{tabular}{|c|c|c|c|c|c|c|c|c|}
\hline \multicolumn{4}{|c|}{ Before PCSK-9 inhibitor injections } & \multicolumn{5}{|c|}{ After PCSK-9 inhibitor injections } \\
\hline & $9 / 17 / 2012$ & $4 / 29 / 2013$ & $4 / 12 / 2014$ & $3 / 24 / 2015$ & $2 / 16 / 2016$ & $2 / 1 / 2017$ & $11 / 27 / 2017$ & $7 / 12 / 2018$ \\
\hline Cholesterol Total (mg/dL) & 235 & 208 & 262 & 198 & 159 & 98 & 166 & 90 \\
\hline WI- (ng/cIL) & 128 & 115 & 152 & 99 & 76 & 22 & 76 & 18 \\
\hline Triglycerides (mg/dL) & 274 & 237 & 286 & 232 & 223 & 164 & 150 & 107 \\
\hline HDL cholesterol (mg/dL) & 52 & 46 & 53 & 60 & 53 & 48 & 56 & 58 \\
\hline LDL/HDL & 2.5 & 4.5 & 4.94 & 3.3 & 0.7 & 2.18 & 1.36 & 0.31 \\
\hline
\end{tabular}

\section{Conclusion}

PCSK-9 inhibitor lowers LDL cholesterol by inhibiting the degradation of LDL receptors (LDLR) and permitting LDLR to recycle back to the liver cell surface. It revealed to regress atherosclerotic plaque in carotid artery stenosis. It may be useful in elderly patients with high risk of perioperative complications as an alternative medical therapy to invasive procedures such as CEA and CAS. Further studies are needed to validate PCSK9 inhibitor as a potential medical treatment for revascularization of carotid artery stenosis.

\section{Acknowledgments}

None.

\section{Funding details}

None.

\section{Conflict of Interests}

The author declares there is no conflict of interest.

\section{References}

1. Benjamin EJ, Virani SS, Callaway CW, et al. Heart disease and stroke statistics - 2018 Update: A report from the American Heart Association. Circulation. 2018;137:e270-e308.

2. Flaherty ML, Kissela B, Khoury JC, et al. Carotid artery stenosis as a cause of stroke. Neuroepidemiology. 2013;40(1):36-41.
3. De Weerd M, Greving JP, de Jong AWF, et al. Prevalence of asymptomatic carotid artery stenosis according to age and sex. Systemic review and metaregression analysis. Stroke. 2009;40(4):1105-1113.

4. Brott TG, Halperin JL, Abbara S, et al. Guideline on the management of patients with extracranial carotid and vertebral artery disease. American College of Cardiology Foundation. 2011. p. 1-51.

5. Ricotta JJ, AbuRahman A, Ascher E, et al. Updated society for vascular surgery guidelines for management of extracranial carotid disease. $J$ Vasc Surg. 2011;54(3):e1-e31.

6. De Rango P, Parlani G, Verzini F, et al. Long-term prevention of stroke: a modern comparison of current carotid stenting and carotid endarterectomy. J Am Coll Cardiol. 2011;57(6):664-671.

7. Boulanger M, Cameliere L, Felgueiras R, et al. Periprocedural myocardial infarction after carotid endarterectomy and stenting: Systemic review and meta-analysis. Stroke. 2015;46(10):2843-2848.

8. Brott TG, Hobson RW, Howard G, et al. Stenting versus endarterectomy for treatment of carotid-artery stenosis. N Eng J Med. 201;363:11-23.

9. Kaufman TM, Duell PB, Prunell JQ, et al. Application of PCSK9 inhibitors in Practice: Challenges and Opportunities. Circ Res. 2017;121(5):499-501.

10. Nicholls SJ, Puri R, Anderson T, et al. Effect of evolocumab on progression of coronary disease in statin-treated patients. JAMA. 2016;316(22):2373-2384.

11. Bonati LH, Gregson J, Dobson J, et al. Restenosis and risk of stroke after stenting or endarterectomy for symptomatic carotid stenosis in the International Carotid Stenting Study (ICSS): secondary analysis of a randomized trial. Lancet Neurol. 2018;17:587-596. 
12. Toklu B, Amirian J, Giugliano RP. Current indications, cost, and clinical use of anti-PCSK9 monoclonal antibodies. Am Coll Cardiol. 2016.

13. Moore WS, Barnett $\mathrm{HJ}$, Beebe $\mathrm{HG}$, et al. Guidelines for carotid endarterectomy. A multidisciplinary consensus statement from the Ad Hoc Committee, American Heart Association. Circulation. 1995;26(1):188-201.

14. Biller J, Feinberg WM, Castaldo JE, et al. Guidelines for carotid endarterectomy: a statement for healthcare professionals from a Special Writing Group of the Stroke Council, American Heart Association. Circulation. 1998;97(5):501-509.

15. International Carotid Stenting Study investigators, Ederle J, Dobson J et al. Carotid artery stenting compared with endarterectomy in patients with symptomatic carotid stenosis (International Carotid Stenting Study): an interim analysis of a randomised controlled trial. Lancet. 2010;375(9719):985-997.

16. Brott TG, Hobson RW, Howard G, et al. Stenting versus endarterectomy for treatment of carotid-artery stenosis. N Engl J Med. 2010;363:11-23.

17. Wu TY, Anderson NE, Barber PA. Neurological complications of carotid revascularisation. J Neurol Neurosurg Psychiatry. 2012;83(5):543-550.

18. Hill MD, Brooks W, Mackey A, et al. Stroke after carotid stenting and endarterectomy in the Carotid Revascularization Endarterectomy versus Stenting Trial (CREST). Circulation. 2012;126(25):3054-3064.

19. Kumamaru H, Jalbert JJ, Nguyen LL, et al. Surgeon case volume and 30-day mortality after carotid endarterectomy among contemporary medicare beneficiaries: before and after national coverage determination for carotid artery stenting. Stroke. 2015;46(5):1288-1294.

20. Hussain MA, Mamdani M, Tu JV, et al. Impact of Clinical Trial Results on the Temporal Trends of Carotid Endarterectomy and Stenting From 2002 to 2014. Stroke. 2016;47(12):2923-2930.

21. Meschia JF, Hopkins LN, Altafullah I, et al. Time From Symptoms to Carotid Endarterectomy or Stenting and Perioperative Risk. Stroke. 2015;46(12):3540-3542.

22. De Rango P, Brown MM, Chaturvedi S, et al. Summary of Evidence on Early Carotid Intervention for Recently Symptomatic Stenosis Based on Meta-Analysis of Current Risks. Stroke. 2015;46(12):3423-436.

23. Henry M, Gopalakrishnan L, Rajagopal S, et al. Bilateral carotid angioplasty and stenting. Catheter Cardiovasc Interv. 2005;64(3):275282.

24. Mathur A, Roubin GS, Iyer SS, et al. Predictors of stroke complicating carotid artery stenting. Circulation. 1998;97(13):1239-1245. 